\title{
Non-linear and Randomized Pricing for Distributed Management of Flexible Loads
}

\author{
Dimitrios Papadaskalopoulos, Member, IEEE, and Goran Strbac, Member, IEEE
}

\begin{abstract}
Price-based management of distributed energy resources (DER) within microgrids is gaining continuously grounds due to scalability and pri vacy limitations of centralized architectures. However, the concentration of flexible loads' response to the lowest-priced periods yields inefficient solutions. A previously proposed measure imposing a flexibility restriction on flexible loads might raise acceptability and feasibility concerns by the users. This paper develops a novel, fully price-based approach where this hard restriction is replaced by a soft, nonlinear price signal. This signal is customized to the operating properties of the different flexible load types by penalizing the square of the demand and the duration of cycle delay of loads with continuously adjustable power levels and deferrable cycles respectively. This approach is shown to produce more efficient solutions than the flexibility restriction measure, for both types of loads. For the latter type, randomization of the non-linear prices brings additional benefits, especially in low operating diversity cases. These contributions are supported by case studies on a microgrid test system, with electric vehicles and wet appliances used as representative examples of the above flexible load types.
\end{abstract}

Index Terms-Dual decomposition, flexible loads, microgrids, price-based management, randomization.

\section{NOMENCLATURE}

\section{A. Distributed Microgrid Management Mechanism}

$r \quad$ Index of iterations

$t^{\text {res }}$ Length of time period in hours

$t \in T$ Index and set of time periods

$n \in N$ Index and set of network nodes

$l \in L \quad$ Index and set of network lines

$i \in I \quad$ Index and set of DER in the mic rogrid

$I_{n} \quad$ Set of DER in the mic rogrid connected at node $n$

$C_{i}(\cdot) \quad$ Cost / disutility function of DER $i$

$F_{i} \quad$ Feasible operation domain of DER $i$

$\boldsymbol{p}_{\boldsymbol{i}}^{\boldsymbol{d e r}, \boldsymbol{r}}$ Vector of active power injections $p_{i, t}^{\text {der,r }}$ of DER $i$ at time period $t$ and iteration $r$

$\boldsymbol{q}_{\boldsymbol{i}}^{\boldsymbol{d e r}, \boldsymbol{r}}$ Vector of reactive power injections $q_{i, t}^{\text {der,r }}$ of DER $i$ at time period $t$ and iteration $r$

$\boldsymbol{p}_{\boldsymbol{n}}^{\boldsymbol{n} e \boldsymbol{t}, \boldsymbol{r}}$ Vector of active power injections $p_{n, t}^{\text {net, } r}$ at node $n$, time period $t$ and iteration $r$

$\boldsymbol{q}_{n}^{\boldsymbol{n} e t, \boldsymbol{r}}$ Vector of reactive power injections $q_{n, t}^{\text {net,r }}$ at node $n$, time period $t$ and iteration $r$

The authors are with the Department of Electrical and Electronic Engineering, Imperial College London, London, U.K. (email: d.papadaskalopoulos08@imperial.ac.uk; g.strbac@imperial.ac.uk) $\boldsymbol{e}_{\boldsymbol{n}}^{\boldsymbol{p}, \boldsymbol{r}} \quad$ Vector of active power imbalances $e_{n, t}^{p, r}$ at node $n$, time period $t$ and iteration $r$

$\boldsymbol{e}_{\boldsymbol{n}}^{\boldsymbol{q}, \boldsymbol{r}}$ Vector of reactive power imbalances $e_{n, t}^{q, r}$ at node $n$, time period $t$ and iteration $r$

$\boldsymbol{v}_{\boldsymbol{t}}^{\boldsymbol{r}} \quad$ Vector of voltage magnitudes $v_{n, t}^{r}$ at node $n$, time period $t$ and iteration $r$

$\boldsymbol{\delta}_{\boldsymbol{t}}^{\boldsymbol{r}} \quad$ Vector of voltage angles $\delta_{n, t}^{r}$ at node $n$, time period $t$ and iteration $r$

$s_{l, t}^{s e n} \quad$ Apparent power flow leaving the reference sending node on line $l$ and time period $t$

$s_{l, t}^{r e c}$ Apparent power flow reaching the reference receiving node on line $l$ and time period $t$

$\underline{v}, \bar{v} \quad$ Minimu $m$ and maximu $m$ allowable voltage magnitude

$\bar{s}_{l} \quad$ Thermal capacity of line $l$

$\lambda_{n}^{r} \quad$ Vector of active power prices $\lambda_{n, t}^{r}$ at node $n$, time period $t$ and iteration $r$

$\boldsymbol{\mu}_{n}^{r} \quad$ Vector of reactive power prices $\mu_{n, t}^{r}$ at node $n$, time period $t$ and iteration $r$

\section{B. Flexible Loads' Price Response Sub-Problems}

$P_{t}^{e v} \quad$ Active power de mand of EV at time period $t$

$P^{\max }$ Maximu $m$ charging rate of $\mathrm{EV}$

$E_{t} \quad$ Energy in EV battery at the end of time period $t$

$E^{\min }$ Minimum energy level in EV battery

$E^{\max }$ Maximu m energy level in EV battery

$E_{t}^{d r} \quad$ Energy requirements of EV for driving purposes at time period $t$

$\eta^{\text {ch }} \quad$ Charging efficiency of EV battery

$T^{g r} \quad$ Set of time periods that EV is connected to the grid

$p f^{e v} \quad$ Power factor of EV charging

$\tau \quad$ Index of steps of the WA cycle

$P_{\tau}^{w a} \quad$ Active power de mand at step $\tau$ of the WA cycle

$T^{\text {dur }}$ Duration of WA cycle

$\tau^{\text {act }}$ Activation time period of WA cycle

$\tau^{\text {in }} \quad$ Initiation time period of WA cycle

$\tau^{\text {ter }}$ Latest termination time period of WA cycle

$d^{\max }$ Maximum delay limit of WA cycle

$p f^{w a}$ Power factor of WA

\section{INTRODUCTION}

\section{A. Motivation}

Aundamental feature of the emerg ing Smart Grid concept Ainvolves the integration of a large number of s mall-scale distributed energy resources (DER), including various types of flexible loads, distributed generators and energy storage units, in distribution networks. Microgrids are generally 
defined as clusters of such DER at different voltage levels of distribution networks, operated as a single autonomous entity, either interconnected or isolated from the main grid [1]. A principal functionality of the microgrid is the optimal active and reactive power management of its DER in order to satisfy economic and technical objectives and constraints of both individual DER and the microgrid as a whole.

Under the traditional centralized management paradigm [2], the local controllers (LC) of DER submit their economic and technical parameters to the microgrid central controller (MGCC), and the latter solves a global AC optimal power flow (OPF) problem and realizes the optimal solution by sending according dispatch signals to the LC. Such centralized approaches however yield communication and computational scalability limitations, as they involve transmission of a large number of complex operational parameters to the MGCC and they require the latter to solve an optimization problem with a vast number of decision variables and constraints Furthermore, they raise privacy concerns by the DER owners who are not generally willing to disclose private information and be directly controlled by external entities [3].

In view of these challenges, an alternative management approach is required, optimally coordinating DER without knowledge of their specific properties by a central entity. Following dual decomposition principles [4], authors in [5] present a distributed, price-based management architecture, involving a two-level iterative process. At the local level, individual DER solve independently their own economic surplus maximization problems for given nodal active and reactive power prices. At the global level, the central coordinator updates these prices in an effort to drive DER responses to the optimal global solution. In [6], this approach is extended to a multiple time-period framework to capture the inter-te mporal operational properties of different DER.

However, this mechanism is not guaranteed to reach a feasible solution, with respect to nodal power balance constraints, if the DER surplus maximization problems are not strictly convex. Strict convexity does not realistically hold for flexible loads, the objective of which is the minimization of their payment, mathe matically expressed through a linear -non strictly convex- objective function.

In an effort to address this complication, authors in [7] outline a heuristic approach; after each iteration, generators' production and loads' consumption is increased or decreased according to a predetermined priority list until power balance constraints are satisfied. However, details on how such priority adjustments should be formed to avoid violation of the operating constraints of DER and achieve near-optimal solutions, are not provided. Authors in [8] calculate after each iteration the average of the DER responses in all previous iterations (primal averaging), and show asymptotic convergence of these averages to a feasible and optimal solution in the limit of iterations approaching infinity. Despite the theoretical significance of this contribution, the interest in practical applications lies in termination in a finite and acceptable (in terms of communication and computational requirements) number of iterations, which the primal averaging technique cannot guarantee [9].

This infeasibility complication is resolved in [10] by fixing the price responses of such non strictly convex DER for part of the coordination process, where iterations are carried out with the strictly convex DER only. However, the same paper has also demonstrated that an optimality challenge emerges. Flexible loads' response is discontinuously concentrated at the lowest-priced periods of the coordination horizon, creating significant new demand peaks and thus yielding inefficient solutions. In order to address this challenge, the authors proposed the perturbation of the flexible loads' response through a relative flexibility restriction. In [10] where flexible loads with continuously adjustable power levels are considered, this flexibility restriction is formed as a maximum power demand limit. In [11], the authors propose a flexibility restriction for flexible loads whose operating constraints do not allow the continuous adjustment of their power demand levels but the deferral of their fixed operation cycles, in the form of a maximu m cycle delay limit.

Imposing a flexibility restriction however may not be deemed acceptable by the users of flexible loads, as they may consider it as a direct intervention of an external entity in the control of their assets. Furthermore, depending on the size of this restriction and the properties of the loads, the satisfaction of their operating constraints could become infeasible; this could be the case for example, if the restricted maximum power demand limit of a load is lower than its total energy requirements divided by the time it is connected to the grid and can acquire this energy.

\section{B. Contributions}

Driven by these acceptability and feasibility challenges, this paper develops a novel, fully price-based approach to tackle the flexible demand response concentration effect, where the hard flexibility restriction is replaced by a soft, non-linear price signal, penalizing the extent of flexibility utilized by the flexible loads. This signal is customized to the particular operating characteristics of the different flexible load types, by penalizing the square of the power demand and the duration of the cycle delay of continuously adjustable and deferrable cycle loads respectively.

Beyond tackling the above acceptability and feasibility challenges, this non-linear pricing approach is demonstrated to outperform the flexibility restriction approach of [10] in flattening the total demand profile and thus producing high quality solutions, for both types of loads. For deferrable cycle loads, it is shown that employment of uniform flexibility restrictions or non-linear prices is not sufficient in achieving efficient solutions, especially if the operating diversity of these loads is low. In order to deal with such cases, a simple approach for the randomization of the non-linear prices is proposed. These contributions are supported by case studies on a microgrid test system, with smart charging electric vehicles (EV) and wet appliances (WA) with delay functionality used as representative examples of the two above flexible load types, and different scenarios investigated regarding their penetration, flexibility and diversity. 


\section{C.Paper Structure}

The rest of this paper is organized as follows. Section II outlines the distributed microgrid management mechanism developed in [10]. Section III formulates the price response sub-problems of the two examined flexible demand technologies. Section IV details the proposed non-linear and randomized pricing approaches. Case studies and illustrative results and presented in Section V. Finally, Section VI discusses conclusions and future extensions of this work.

\section{DIST RIBUTED MICROGRID MANAGEMENT MECHANISM}

The microgrid management problem is formulated as a social welfare maximization problem (1), assuming that $C_{i}$ constitutes a cost function if DER $i$ is a generator and a disutility function if DER $i$ is a load. This problem is subject to the nodal active (2) and reactive (3) power balance constraints, the voltage (4) and thermal (5)-(6) limits of the distribution network, and the individual operational constraints of the DER (7). As in [10], the responsible entity for microgrid management is termed microgrid market operator (MGMO).

$\min f=\min _{\left\{\boldsymbol{p}_{\boldsymbol{i}}^{\text {der }}\right\},\left\{\boldsymbol{q}_{\boldsymbol{i}}^{\text {der }}\right\},\left\{\boldsymbol{v}_{\boldsymbol{t}}\right\},\left\{\boldsymbol{\delta}_{\boldsymbol{t}}\right\}} \sum_{i \in I} C_{i}\left(\boldsymbol{p}_{\boldsymbol{i}}^{\text {der }}, \boldsymbol{q}_{\boldsymbol{i}}^{\text {der }}\right)$

Subject to:

$e_{n, t}^{p} \equiv p_{n, t}^{n e t}\left(\boldsymbol{v}_{\boldsymbol{t}}, \boldsymbol{\delta}_{\boldsymbol{t}}\right)-\sum_{i \in I_{n}} p_{i, t}^{d e r}=0, \forall t \in T, \forall n \in N$

$e_{n, t}^{q} \equiv q_{n, t}^{n e t}\left(\boldsymbol{v}_{\boldsymbol{t}}, \boldsymbol{\delta}_{\boldsymbol{t}}\right)-\sum_{i \in I_{n}} q_{i, t}^{d e r}=0, \forall t \in T, \forall n \in N$

$\underline{v} \leq v_{n, t} \leq \bar{v}, \forall t \in T, \forall n \in N$

$s_{l, t}^{\text {sen }}\left(\boldsymbol{v}_{\boldsymbol{t}}, \boldsymbol{\delta}_{\boldsymbol{t}}\right) \leq \bar{s}_{l}, \forall t \in T, \forall l \in L$

$s_{l, t}^{r e c}\left(\boldsymbol{v}_{\boldsymbol{t}}, \boldsymbol{\delta}_{\boldsymbol{t}}\right) \leq \bar{s}_{l}, \forall t \in T, \forall l \in L$

$\left(\boldsymbol{p}_{\boldsymbol{i}}^{\text {der }}, \boldsymbol{q}_{\boldsymbol{i}}^{\text {der }}\right) \in F_{i}, \forall i \in I$

The primal problem (1)-(7) is solved indirectly by solving its Lagrangian dual problem (8), where $\varphi$ is the dual function and $L$ is the Lagrangian function (9) of the problem, derived by relaxing constraints (2) and (3) through Lagrangian mu ltip lier vectors $\boldsymbol{\lambda}_{\boldsymbol{n}}$ and $\boldsymbol{\mu}_{\boldsymbol{n}}$ respectively.

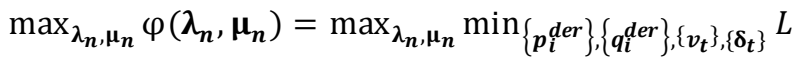

Inner minimization subject to: (4)-(7)

$L=\sum_{i \in I} C_{i}\left(\boldsymbol{p}_{\boldsymbol{i}}^{\text {der }}, \boldsymbol{q}_{\boldsymbol{i}}^{\boldsymbol{d e r}}\right)+\sum_{t \in T} \sum_{n \in N}\left(\lambda_{n, t} e_{n, t}^{p}+\mu_{n, t} e_{n, t}^{q}\right)$

The dual problem (8) is decomposed to independent subproblems -one corresponding to each DER (10) and one corresponding to the distribution network (11) (assumed that is solved by the MGMO)- coordinated iteratively by a $\boldsymbol{\lambda}_{\boldsymbol{n}}$ and $\boldsymbol{\mu}_{\boldsymbol{n}}$ update until $\varphi$ is maximized. This decomposition scheme yields a two-level market-based management mechanis $\mathrm{m}$, with the elements of $\boldsymbol{\lambda}_{\boldsymbol{n}}$ and $\boldsymbol{\mu}_{\boldsymbol{n}}$ representing active and reactive power prices respectively, at each node and time period. At the local level and for given prices, the LC of each DER $i \in I_{n}$ solves independently their surplus maximization problem (10) and the MGMO solves independently the congestion surplus maximization problem (11). At the global level, the MGMO updates the prices in an effort to gradually maximize $\varphi$; subgradient [6]-[8] or cutting-plane algorith ms [12] are employed in the majority of relevant works for this update.

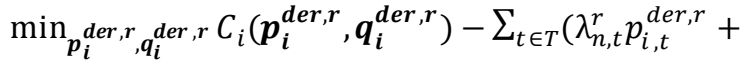
$\mu n, t r q i, t d e r, r) \quad$ Subject to: (pider,r,qider,r) $\in F i$

$\min _{v_{t}^{r}, \boldsymbol{\delta}_{t}^{r}} \sum_{t \in T} \sum_{n \in N}\left(\lambda_{n, t}^{r} p_{n, t}^{n e t, r}\left(\boldsymbol{v}_{\boldsymbol{t}}^{r}, \boldsymbol{\delta}_{t}^{r}\right)+\mu_{n, t}^{r} q_{n, t}^{n e t, r}\left(\boldsymbol{v}_{\boldsymbol{t}}^{r}, \boldsymbol{\delta}_{\boldsymbol{t}}^{r}\right)\right)$

Subject to: (4)-(6)

The optimal solution of the dual problem $\varphi^{*}$ is guaranteed to coincide with the optimal solution of the primal problem $f^{*}$ and satisfy the relaxed constraints (2)-(3), only if the DER sub-problems (10) are strictly convex [5]-[6]. In [10]-[11], we identified non strict convexities in the sub-problems of both continuously adjustable and deferrable cycle loads, as sociated with their linear payment minimization objective function and -specifically for the latter type- their discrete operation domain. In [10], we demonstrated through simple examples that the above iterative mechanism cannot generally satisfy the power balance constraints (2)-(3) due to these non strict convexities, irrespectively of the multip liers' update algorithm employed and the number of iterations executed.

In order to resolve this infeasibility complication, we proposed the management mechanis $m$ illustrated in Fig. 1 [10]. In the external loop, the LC of all DER determine their price responses by solving their sub-problems, and the MGMO solves the network sub-problem, evaluates $\varphi$, and updates $\lambda_{n}$ and $\boldsymbol{\mu}_{n}$ to gradually maximize $\varphi$. In the internal loop, the responses of the flexible loads are fixed to their respective values at the latest external iteration, and iterations are carried out with the strictly convex DER only. This simple technique eliminates the effect of the flexible loads' nonstrict-convexities, and therefore allows the internal loop to reach a solution satisfying the power balance constraints, at which point it is terminated.

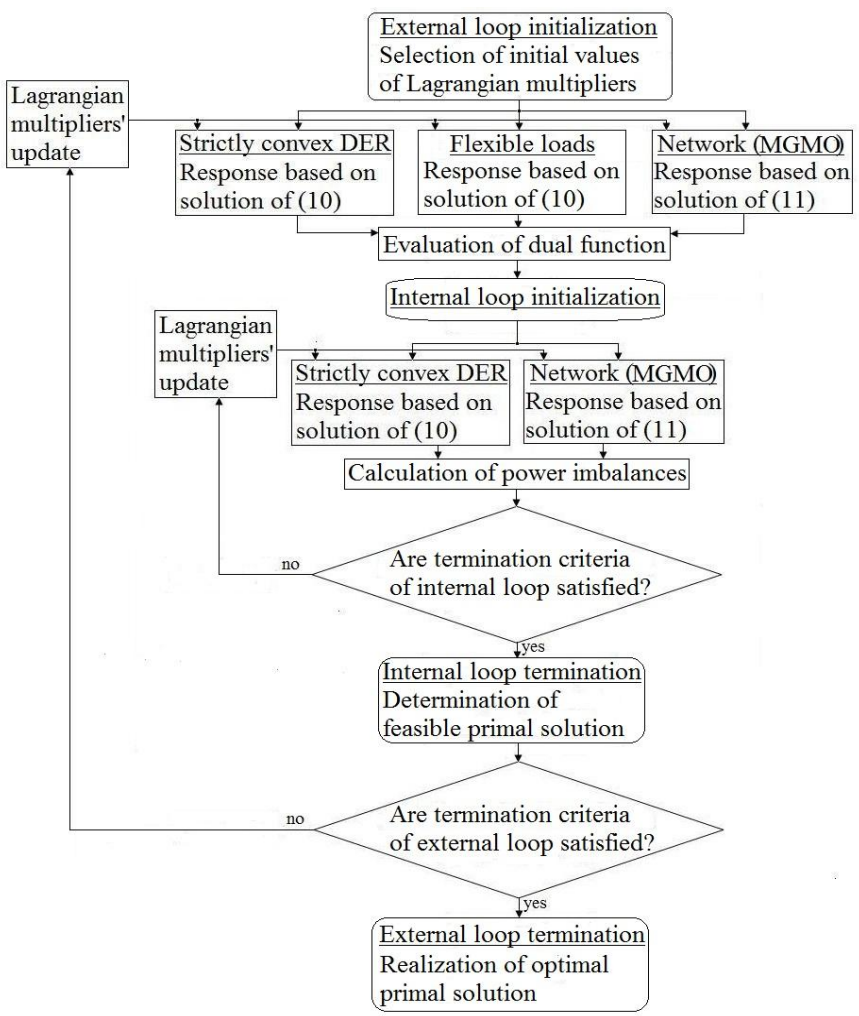

Fig. 1. Flowchart of distributed microgrid management mechanism 
The feasible primal solution $f^{r}$ produced at the external iteration $r$ is not guaranteed to be optimal. However, a quantitative indication of its optimality is provided by the weak duality theorem [4], according to which any dual solution $\varphi^{r}$ satisfies $\varphi^{r} \leq f^{*}$. Given that $f^{r} \geq f^{*}$, the optimality of $f^{r}$ is indicated by the relative duality gap ( $\left.R D G\right)$ (12) [12], which constitutes an upper bound of the distance between $f^{r}$ and $f^{*}$. The proposed mechanis $\mathrm{m}$ is terminated when the $R D G$ of the minimum available $f^{r}$ is lower than a pre-determined tolerance $\varepsilon$ or a maximum number of external iterations $R$ has been carried out.

$R D G=\left(f^{r}-\max \left(\varphi^{r}\right)\right) / \max \left(\varphi^{r}\right)$

\section{PRICE RESPONSE SUB-PROBLEMS OF FLEXIBLE LOADS}

Two types of flexible loads are examined, both requiring an amount of energy over a temporal interval specified by their users. For the first type, the power demand level can be continuously adjusted up to a maximum rate. Smart charging EV [13], which need to obtain the energy required for the desired journeys over the interval they are connected to the grid, are employed as a representative examp le of this type.

The operation of the second type is based on the execution of user-called cycles which comprise a sequence of phases occurring at a fixed order with generally fixed duration and fixed power demand, that cannot be altered; their flexibility involves the deferability of these cycles up to a maximum delay limit set by their users. WA (e.g. dishwashers and washing machines) with delay functionality [14] constitute a representative example of this type.

\section{A. EV with Smart Charging Capability}

Assuming a constant, uncontrollable power factor, the EV price response sub-problem is formulated as:

$\min _{\left\{P_{t}^{e v}\right\}} \sum_{t \in T}\left(\lambda_{t}+\mu_{t} * \tan \cos ^{-1} p f^{e v}\right) * P_{t}^{e v}$

Subject to:

$E_{t}=\eta^{c h} * P_{t}^{e v} * t^{r e s}+E_{t-1}-E_{t}^{d r}, \forall t \in T$

$E^{\min } \leq E_{t} \leq E^{\max }, \forall t \in T$

$0 \leq P_{t}^{e v} \leq P_{t}^{\max }, \forall t \in T$

$P_{t}^{\max }=\left\{\begin{array}{cc}P^{\max } & \text { if } t \in T^{g r} \\ 0 & \text { otherwise }\end{array}\right.$

$E_{0}=E_{|T|}$

Constraint (14) expresses the energy balance in the EV battery. Constraint (15) corresponds to the battery's maximum depth of discharge and state of charge ratings. Constraints (16)-(17) represent the limit of the battery's power input, which depends on the maximum charging rate of the battery and on whether the EV is connected to the grid. The EV demand redistributing ability is spread beyond the temporal horizon of the microgrid management problem; for the sake of simplicity, the battery energy content at the start and the end of the horizon are assumed equal (18).

\section{B. WA with Delay Functionality}

Without loss of generality, each WA is assumed to be activated by its users once during the temporal horizon of the problem. Also assuming a constant, uncontrollable power factor, the WA price response sub-problem is formulated as:

$\min _{t^{i n} \in \mathbb{N}} \sum_{\tau=1}^{T^{d u r}}\left(\lambda_{t^{i n}+\tau-1}+\mu_{t^{i n}+\tau-1} * \tan \cos ^{-1} p f^{w a}\right) *$

Ptwa

Subject to:

$t^{\text {act }} \leq t^{\text {in }} \leq t^{\text {act }}+d^{\text {max }}$

where $d^{\text {max }}=1+t^{\text {ter }}-t^{a c t}-T^{d u r}$

Constraints (20)-(21) express the users' requirements; the cycle cannot be initiated before the activation time of the appliance (since users need to load and switch on the appliance before the latter initiates its cycle) and cannot be terminated after the latest time desired by the users. In order to capture out-of-horizon effects, a periodic continuation is assumed, implying that the WA assume $\lambda_{t+|T|}=\lambda_{t}$ and $\mu_{t+|T|}=\mu_{t}$ and the demand of WA migrating towards / away from period $t+|T|$ is added to / subtracted from the total demand at period $t$.

\section{MEASURES Against DEMAND ReSPONSE CONCENTRATION}

Although the mechanism of Fig. 1 is guaranteed to produce feasible solutions $f^{r}$, the same does not apply on their optimality. As demonstrated in [10]-[11], flexible loads' response is discontinuously concentrated at the time periods with the lowest prices at $r$ due to their non-strict-convexities, creating significant new demand peaks and thus yielding ineffic ient solutions.

\section{A. Flexibility Restriction}

Driven by the observation that the size of this concentration effect is enhanced when the loads' flexibility extent is higher, we previously proposed the application of a relative flexibility restriction $\omega$, which represents the fraction of available fle xibility that can be utilized by the loads [10]-[11]. For loads with continuously adjustable power levels, $\omega$ represents their maximum power limit as a fraction of the respective nominal one. Given that a larger power limit enables such loads to acquire larger proportion of their energy requirements at the lowest-priced period, the application of $\omega$ tends to limit high demand levels from each load. For s mart charging EV, the introduction of $\omega$ transforms constraint (16) to (22):

$0 \leq P_{t}^{e v} \leq \omega * P_{t}^{\max }, \forall t \in T$

For loads with deferrable cycles, a different approach is required, since their power demand levels are fixed and cannot be modified; $\omega$ represents the maximum cycle delay limit as a fraction of the respective limit set by the users [11]. Given that the delay limit determines the range of the time window within which these loads can execute their cycles, and that different loads are characterized by different activation times, the application of $\omega$ tends to limit the nu mber of loads that can execute their cycles at the same low-priced periods. For WA with delay functionality, the introduction of $\omega$ transforms constraint (20) to (23):

$t^{a c t} \leq t^{\text {in }} \leq t^{a c t}+\omega * d^{\text {max }}$ 


\section{B. Non-Linear Pricing}

Imposing such flexibility restrictions may not be deemed acceptable by the users of the flexible loads, as they may consider it as a direct intervention of an external entity in the control of their assets. Furthermore, depending on the value of $\omega$ and the properties of the loads, the satisfaction of their operating constraints could be threatened. For a smartcharging EV for example, if $\omega * \eta^{\text {ch }} * P^{\max } *\left|T^{g r}\right| * t^{r e s}<$ $\sum_{t} E_{t}^{d r}$, then its response sub-problem becomes in feasible.

In order to circumvent these challenges, the alternative approach proposed in this paper replaces this hard flexibility restriction by a soft non-linear price signal $\alpha$, effectively penalizing the extent of flexibility utilized by the loads.

For loads with continuously adjustable power, $\alpha$ penalizes the square of the power demand and thus indirectly limits high demand levels from each load. Such a quadratic price follows the inclining block rate pricing concept [15], according to which the marginal price increases with the size of the consumed quantity. For smart charging EV, the introduction of $\alpha$ transforms their objective function (13) to (24):

$\min _{\left\{P_{t}^{e v}\right\}} \sum_{t \in T}\left(\lambda_{t}+\mu_{t} * \tan \cos ^{-1} p f^{e v}\right) P_{t}^{e v}+\alpha\left(P_{t}^{e v}\right)^{2}$

Apart from the above acceptability and feasibility advantages, quadratic pricing exhibits an optimality advantage over the flexibility restriction approach. With the latter, the response sub-problem remains linear and thus the solution always involves demand equal to the new maximum power limit at the periods with the lowest prices. With the former approach on the other hand, the objective function becomes quadratic, the optimal response can admit a larger number of values in the interior of the feasible operation domain, and thus a better total demand flattening effect can be achieved.

This is clarified through a single-node, three-hour example, where the microgrid includes inflexible demand $\boldsymbol{D}^{\text {inf }}=$ $[1,2,3] k W$, a generator with quadratic cost function, and a continuously adjustable flexible load with total energy requirements $E=6 \mathrm{kWh}$ and maximum power $P^{\max }=6 \mathrm{~kW}$. The optimal solution of the microgrid management problem involves $\boldsymbol{p}=[3,2,1] \mathrm{kW}$ for the flexible load, leading to a completely flattened total demand profile $\boldsymbol{D}^{\boldsymbol{t} \boldsymbol{t} t}=[4,4,4] \mathrm{kW}$.

Assuming that the linear prices at a specific external iteration of the distributed management mechanis $\mathrm{m}$ are $\boldsymbol{\lambda}=[1,2,3] p / k W h$, it can be observed that the flexibility restriction approach cannot realize such a response from the flexible load, irrespectively of the value of $\omega \in(0,1]$. For example, $\omega=0.33$ gives $\boldsymbol{p}=[2,2,2] \mathrm{kW}, \omega=0.4$ gives $\boldsymbol{p}=[2.4,2.4,1.2] \mathrm{kW}, \omega=0.5$ gives $\boldsymbol{p}=[3,3,0] \mathrm{kW}$ and $\omega=0.6$ gives $\boldsymbol{p}=[3.6,2.4,0] \mathrm{kW}$. The above response could only be achieved if time-specific flexibility restrictions were allowed (with values $\omega_{1}=0.5$ and $\omega_{2}=0.33$ ). However, the complexity of setting suitable time-specific values would be dramat ically higher. On the other hand, the proposed quadratic pricing approach can realize the above response without such comple xity, with a uniform quadratic price $\alpha=0.5 \mathrm{p} / \mathrm{kWh}^{2}$.

For loads with deferrable cycles, $\alpha$ penalizes the duration of the cycle delay, and given that different loads are characterized by different activation times, it indirectly limits the number of loads that can execute their cycles at the same periods. For WA with delay functionality, the introduction of $\alpha$ transforms their objective function (19) to (25):

$$
\min _{t^{i n} \in \mathbb{N}} \sum_{\tau=1}^{T^{d u r}}\left(\lambda_{t^{i n}+\tau-1}+\mu_{t^{i n}+\tau-1} * \tan \cos ^{-1} p f^{w a}\right) *
$$

Ptwa $+\alpha * d \max$

According to the above discussion, a critical assumption for the effectiveness of both flexibility restriction and non-linear pricing approaches in the case of loads with deferrable cycles is that different loads exhibit different activation times. As such operating diversity gets lower, the performance of both above approaches gets worse. This can be better understood by considering the extreme example where all deferrable cycle loads at a specific node have identical operating parameters. In this case, irrespectively of the value of $\omega$ or $\alpha$, all loads will initiate their cycle at the same time period and the concentration effect cannot be avoided.

However, this challenge is partially addressed with the nonlinear pricing approach, as the optimal response of loads connected to different nodes can be diversified due to the different correlation between the size of linear price differentials and the delay price $\alpha$. This is clarified through a two-node, two-hour example, where inflexible demand at the two nodes A and B is $\boldsymbol{D}_{\boldsymbol{A}}^{\boldsymbol{i n f}}=[4,2] \mathrm{kW}$ and $\boldsymbol{D}_{\boldsymbol{B}}^{\boldsymbol{i n f}}=[8,4] \mathrm{kW}$. 6 and 12 identical WA are connected to nodes $\mathrm{A}$ and $\mathrm{B}$ respectively, each characterized by $T^{d u r}=1, P_{1}^{w a}=1 \mathrm{~kW}$, $\tau^{a c t}=1$ and $d^{\max }=1$; in other words each WA can carry out their cycle either at $t=1$ or $t=2$.

Assuming that the linear prices at a specific external iteration of the distributed management mechanis $\mathrm{m}$ are $\boldsymbol{\lambda}_{\boldsymbol{A}}=[4,2] p / k W h$ and $\boldsymbol{\lambda}_{\boldsymbol{B}}=[8,4] p / k W h$, it can be observed that if the distributed management mechanism employs the flexibility restriction approach it can only either: i) allow all WA to shift to the hour with the lowest linear price $t=2$ (with $\omega=1$ ), yielding a total demand profile of $\boldsymbol{D}^{\text {tot }}=$ $[12,24] \mathrm{kW}$ or ii) do not allow this shift (with $\omega<1$ ), yielding a total demand profile of $\boldsymbol{D}^{\boldsymbol{t} \boldsymbol{t} t}=[30,6] \mathrm{kW}$. If the proposed delay pricing approach is employed with $\alpha=3 p / h$, the WA of node A will be incentivized to execute their cycle at $t=1$, given that the cost of shifting $(3 p)$ is higher than the benefit of shifting to the lowest-priced $t=2(2 p)$. On the other hand, the WA of node B will shift their cycle to $t=2$ as the relevant benefit $(4 p)$ is higher than the relevant cost. This diversified response among the two nodes yields a completely flattened demand profile $\boldsymbol{D}^{\text {tot }}=[18,18] \mathrm{kW}$ which corresponds to the optimal solution of the problem.

\section{C.Randomized Non-Linear Pricing}

Based on authors' studies, the extent of deferrable cycle loads' response diversification achieved with uniform nonlinear pricing, due to the analy zed effect of differentiated price variations at the different nodes, is not generally sufficient to tackle the demand concentration effect, especially in cases with low operating diversity of such loads. Therefore, it has been deemed necessary to introduce an additional degree of 
diversity in their response by diversifying the non-linear prices $\alpha$. Since the MGMO does not have any information on the parameters of the flexible loads to drive the specifics of such diversification, a randomization approach is proposed.

A vector $X$ is derived by a random number generator, the size of which is equal to the number of loads in the microgrid and its elements $x_{j}$ take random values following the standard normal distribution (normal distribution with zero mean and unity standard deviation). The randomised non-linear price $\alpha_{j}^{\text {rand }}$ transmitted to the $\mathrm{LC}$ of each of the loads $j$ is given by (26), where $\alpha^{*}$ and $\sigma$ denote respectively the mean value and standard deviation of the distribution of non-linear prices.

$\alpha_{j}^{\text {rand }}=\alpha^{*}+\sigma * x_{j}$

\section{Tuning Measures' Parameters}

As quantitatively demonstrated in Section V, relatively large values of $\omega$ and relatively s mall values of $\alpha$ and $\sigma$ may not sufficiently limit the loads' flexibility to concentrate their demand at the lowest-priced periods, while relatively s mall values of $\omega$ and relatively large values of $\alpha$ and $\sigma$ may limit excessively their flexibility and thus prevent them from shaving the peaks and filling the off-peak valleys of the infle xible demand profile.

Suitable values of $\omega, \alpha$ and $\sigma$ should be employed to achieve an effective trade-off between these two effects, leading to a flatter demand profile and a more efficient solution. As demonstrated in Section V, such suitable values will depend on the correlation between the characteristics of the flexible loads population (number, nominal flexibility and diversity) and the temporal variation of infle xible demand. For a certain inflexible demand profile, a larger number and flexibility and a lower diversity of the flexible loads population generally result in a smaller value of the most suitable $\omega$ and a larger value of the most suitable $\alpha$ and $\sigma$.

According to the approach proposed in [10], the MGMO applies a set $\Omega=\left\{\omega_{k}, k=1,2, \ldots, K\right\}, \mathrm{A}=\left\{\alpha_{k}, k=1,2, \ldots, K\right\}$ or $\mathrm{A}^{\text {rand }}=\left\{\boldsymbol{\alpha}_{\boldsymbol{k}}^{\text {rand }}, k=1,2, \ldots, K\right\}$ (based on sub-Section IV$\mathrm{C}$, each element of the last set is a vector of randomized prices submitted to the different loads, and corresponding to a value $\sigma_{k}$ of the standard deviation) to the flexible loads at each external iteration of the distributed management mechanis $\mathrm{m}$, in order to heuristically search for a suitable value of $\omega, \alpha$ or $\sigma$ respectively. The LC of these loads solve their modified price response sub-problems for each element of the set, and for their (fixed) response corresponding to each element, the MGMO carries out iterations with the strictly convex DER to calculate $K$ alternative feasible solutions $f_{k}^{r}$ of the primal problem. In real implementations, it is envisaged that after gaining some experience, the MGMO will be able to guide the search for an efficient solution without the need to try out a large nu mber of $\omega, \alpha$ and $\sigma$ values.

In cases where the microgrid includes both loads with continuously adjustable power levels and loads with deferrable cycles, application of either identical or differentiated between the two types, $\omega, \alpha$ and $\sigma$ values can be conceived. As quantitatively demonstrated in Section V, the latter approach expands the space of the heuristic search leading to higher quality solutions, at the cost of more complex tuning of the differentiated parameters.

\section{V.CASE STUDIES}

\section{A. Description of Case Studies}

Case studies are carried out on a microgrid test system proposed in [16] (Fig. 2). A day-ahead horizon with hourly resolution is considered for the microgrid management problem. The microgrid contains six micro-generators with quadratic cost functions, the parameters of which are given in [3]. It is assumed that the main grid cannot absorb but can supply power to the microgrid, with the relevant quadratic cost function parameters also given in [3]. Furthermore, the cost function associated with demand curtailment is assumed 10 times higher than the cost function of the most expensive micro-generator.

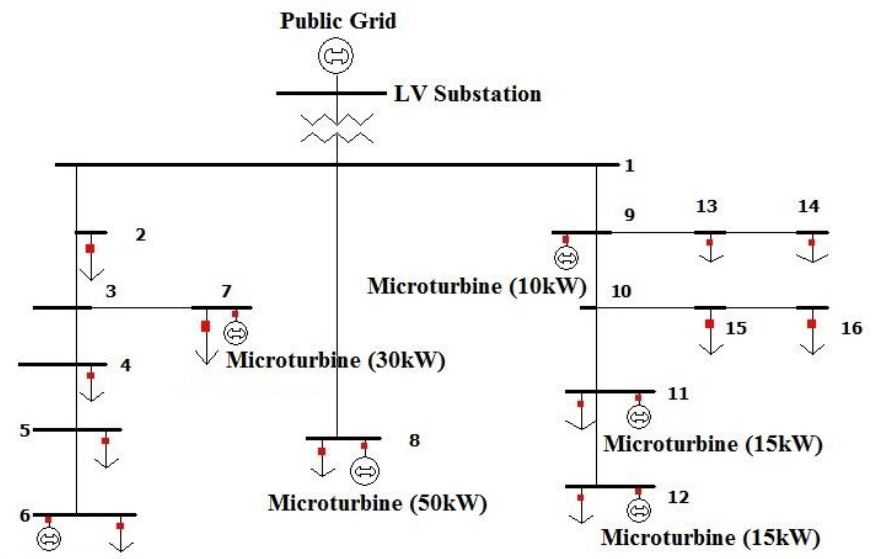

Fuel Cell (30kW)

Fig. 2. Microgrid test system

Different scenarios are investigated regarding the penetration, fle xibility and diversity of smart-charg ing EV and WA with delay functionality in the microgrid. Three EV penetration scenarios (low-LP, medium-MP, high-HP) are examined, with the respective number of EV given in Table I. The total number of EV in each scenario is spread to the different nodes, proportionally to their peak inflexible demand. Furthermore, three EV flexibility scenarios (low-LF, medium-MF, high- HF) are examined, corresponding to different EV maximum power charging rates (Table I). The values of the rest of EV parameters are given in Table II.

TABLE I

EVSCENARIOS

\begin{tabular}{|l|c|c|c|}
\hline & Low & Medium & High \\
\hline Penetration (EV number) & 17 & 34 & 51 \\
\hline Flexibility $\left(P^{\max }\right)$ & $1 \mathrm{~kW}$ & $3 \mathrm{~kW}$ & $6 \mathrm{~kW}$ \\
\hline
\end{tabular}

TABLE II

EV AND WAPARAMETERS

\begin{tabular}{|c|c|c|c|c|}
\hline \multicolumn{2}{|c|}{ EV } & \multicolumn{3}{|c|}{ WA } \\
\hline Parameter & Value & \multirow{2}{*}{ Parameter } & \multicolumn{2}{|c|}{ Value } \\
\cline { 5 - 6 } \cline { 4 - 5 } & $\{1, ., 8,19 \ldots, 24\}$ & & DW & WM \\
\hline$T_{t}^{g r}$ & $7.12 \mathrm{kWh}$ & $T^{\text {dur }}$ & 2 & 2 \\
\hline$E_{t}^{\text {min }}$ & $3 \mathrm{kWh}$ & $P_{1}^{\text {wa }}$ & $0.56 \mathrm{~kW}$ & $0.78 \mathrm{~kW}$ \\
\hline$E^{\text {max }}$ & $15 \mathrm{kWh}$ & $P_{2}^{\text {wa }}$ & $0.63 \mathrm{~kW}$ & $0.11 \mathrm{~kW}$ \\
\hline$\eta^{c h}$ & 0.93 & $p f^{w a}$ & 0.9 & 0.9 \\
\hline$p f^{e v}$ & 0.9 & & & \\
\hline
\end{tabular}


Two different types of WA are considered, namely dishwashers (DW) and washing machines (WM), with the demand profiles of their cycles given in Table II [14]. Three flexibility scenarios (low-LF, medium-MF, high-HF) are examined, corresponding to different maximum delay limits set by the users (Table III). Furthermore, three diversity scenarios (low-LD, medium-MD, high-HD) are examined (Table III). In the HD scenario, the total number of WA in the microgrid (320) is equally divided to DW and WM and their activation times are diversified according to European consumers' surveys [14]. In the MD scenario, the microgrid includes only DW with activation times diversified according to [14], while in the LD scenario the microgrid includes only DW, all activated at the same hour $(t=20)$. The total number of DW and WM in each scenario is spread to the different nodes, proportionally to their peak inflexible demand.

TABLE III

WA SCENARIOS

\begin{tabular}{|l|c|c|c|}
\hline & Low & Medium & High \\
\hline Flexibility $\left(d^{\max }\right)$ & $5 \mathrm{~h}$ & $10 \mathrm{~h}$ & $15 \mathrm{~h}$ \\
\hline Diversity & $\begin{array}{c}\text { Only DW, } \\
\text { uniform } \tau^{a c t}\end{array}$ & $\begin{array}{c}\text { Only DW, } \\
\text { diversified } \tau^{a c t}\end{array}$ & $\begin{array}{c}\text { DW and WM, } \\
\text { diversified } \tau^{a c t}\end{array}$ \\
\hline
\end{tabular}

The distributed management mechanism, in combination with the three measures against demand response concentration, was implemented in MATLAB, and the case studies were carried out on a desktop computer with a $3.33 \mathrm{GHz}$ processor and $12 \mathrm{~GB}$ of RAM. In every case, the multipliers $\lambda_{n}$ and $\boldsymbol{\mu}_{n}$ were initialized to their optimal values in the case where no flexible loads are included in the microgrid. The penalty-bundle algorithm of [10] was employed for updating the multipliers. The termination parameters of the mechanis $\mathrm{m}$ were set to $\varepsilon=1 \%, R=20$, and in every case the relative duality gap termination criterion was satisfied (reached a value below $1 \%$ ) before reaching the maximum number of external iterations. The maximum computation time required for a single case was $387 \mathrm{~s}$.

Suitable values $\omega^{*}, \alpha^{*}$ and $\sigma^{*}$ of the flexibility restriction, non-linear price and standard deviation in each case have been determined by heuristically trying out a range of values, according to the discussion in Section IV-D. Specifically, the trialled values of $\omega$ ranged from 0.1 to 1 with a step of 0.1 , and the values of $\alpha$ and $\sigma$ ranged from 0 to 2 with a step of 0.1 . In the case of randomized non-linear pricing, the most suitable value of the uniform non-linear price was employed as the mean value of the distribution of non-linear prices.

\section{B. Analysis of Cases with Flexible EV}

Fig. 3 illustrates the benefits of the three measures in terms of total cost reduction (with respect to the case where no measure is applied i.e. only the linear prices $\lambda_{n}$ and $\mu_{n}$ are used for coordinating the EV) for different values of their parameters $\omega, \alpha$ and $\sigma$, in the medium penetration - high flexibility scenario. Very large values of $\omega$ and very small values of $\alpha$ do not sufficiently limit the EVs' flexibility to concentrate their demand at the lowest-priced periods, while very small values of $\omega$ and very large values of $\alpha$ limit excessively their flexibility and thus prevent them from shaving the peaks and filling the off-peak valleys of the inflexible demand profile. The most suitable values of these parameters achieve an effective trade-off between these two effects, and the highest benefits; these are $\omega^{*}=0.2$ and $\alpha^{*}=0.5$ for this scenario. In the case of $\mathrm{EV}$, rando mization of the non-linear prices does not bring additional benefits and the most suitable value of the standard deviation is $\sigma^{*}=0$.

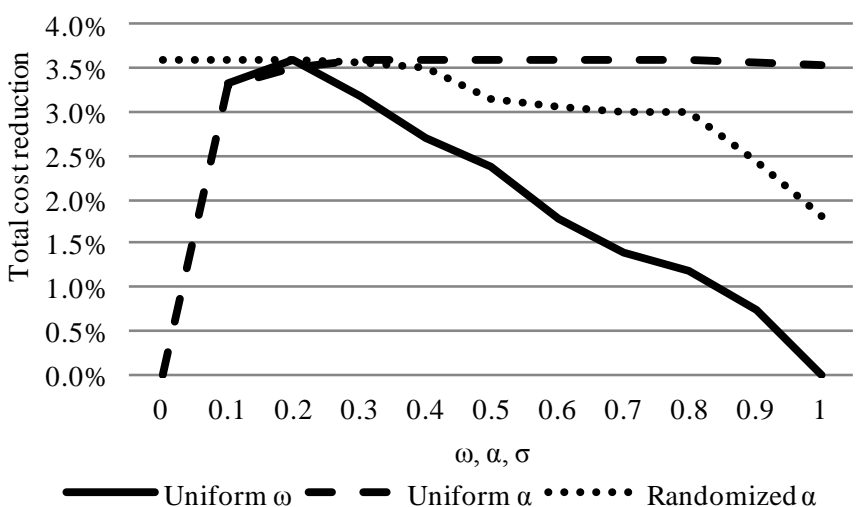

Fig. 3. Benefits of demand response concentration measures for different values of $\omega, \alpha$ and $\sigma$ in medium penetration - high flexibility EV scenario

Table IV presents the most suitable values $\omega^{*}, \alpha^{*}$ and $\sigma^{*}$ of the parameters of the three measures, as well as their benefits, for each of the examined scenarios. As the penetration and flexibility of EV is enhanced, the size and the cost implications of the new peaks created in the case without measures are significantly aggravated; as a result, the benefits of flexibility restriction and non-linear pricing are increased. Such benefits get dramatically higher in the high penetration high flexibility case, as the EV response when no measure is applied activates the minimum voltage constraint at node 7 (exhibiting the highest infle xib le de mand and EV nu mber) and requires expensive demand curtailment. Furthermore, $\omega^{*}$ and $\alpha^{*}$ are decreased and increased respectively, as a more significant restriction/penalization of EV flexibility is required to avoid the demand concentration effect. As mentioned earlier, randomization of the non-linear prices does not bring additional benefits and $\sigma^{*}=0$ holds for every scenario.

TABLE IV

OP TIMAL TUNING AND BENEFTS OF DEMAND RESPONSE CONCENTRATION MEASURES IN EV SCENARIOS

\begin{tabular}{|l|c|c|c|c|c|c|}
\hline Scenario & \multicolumn{2}{|c|}{$\begin{array}{c}\text { Uniform flexibility } \\
\text { restriction }\end{array}$} & \multicolumn{2}{|c|}{$\begin{array}{c}\text { Uniform non-linear } \\
\text { pricing }\end{array}$} & \multicolumn{2}{c|}{$\begin{array}{c}\text { Randomizednon- } \\
\text { linear pricing }\end{array}$} \\
\hline & $\omega^{*}$ & Benefit & $\alpha^{*}$ & Benefit & $\sigma^{*}$ & Benefit \\
\hline LP-LF & 1 & $0.00 \%$ & 0 & $0.00 \%$ & 0 & $0.00 \%$ \\
\hline LP-MF & 0.5 & $0.23 \%$ & 0.2 & $0.25 \%$ & 0 & $0.25 \%$ \\
\hline LP-HF & 0.2 & $0.78 \%$ & 0.2 & $0.89 \%$ & 0 & $0.89 \%$ \\
\hline MP-LF & 1 & $0.00 \%$ & 0.5 & $0.00 \%$ & 0 & $0.00 \%$ \\
\hline MP-MF & 0.4 & $1.25 \%$ & 0.5 & $1.32 \%$ & 0 & $1.32 \%$ \\
\hline MP-HF & 0.2 & $3.59 \%$ & 0.5 & $3.76 \%$ & 0 & $3.76 \%$ \\
\hline HP-LF & 1 & $0.00 \%$ & 1 & $0.01 \%$ & 0 & $0.01 \%$ \\
\hline HP-MF & 0.4 & $2.65 \%$ & 1 & $2.79 \%$ & 0 & $2.79 \%$ \\
\hline HP-HF & 0.2 & $16.28 \%$ & 1 & $16.55 \%$ & 0 & $16.55 \%$ \\
\hline
\end{tabular}

Fig. 4 illustrates the inflexible active demand profile at node 7 , along with the respective total demand profile in the high penetration - medium flexibility scenario, for the cases where no demand response concentration measure is applied, and the flexibility restriction and non-linear pricing measures are applied (with the most suitable values of their parameters $\omega^{*}$ and $\alpha^{*}$ ). In the former case, a very large new peak is created by EV response at the hours with the lowest (linear) prices (46). Both measures prevent the creation of this peak and efficiently distribute the EV demand across the off-peak 
valley. For the reason analyzed in Section IV-B, non-linear pricing yields a flatter demand profile (Fig. 4) and therefore higher total cost reduction benefits (Table IV) than the fle xibility restriction approach.

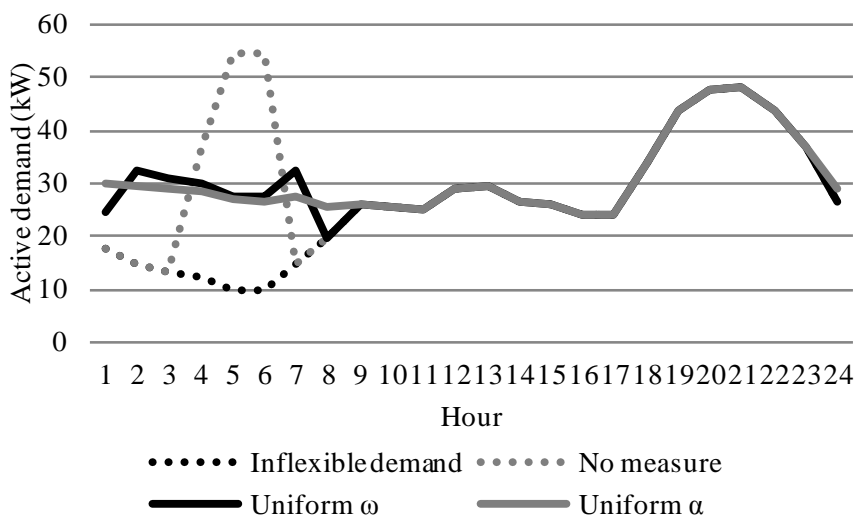

Fig. 4. Active demand profile at node 7 in high penetration - medium flexibility EV scenario

\section{C.Analysis of Cases with Flexible WA}

Fig. 5 illustrates the benefits of the three measures for different values of their parameters $\omega, \alpha$ and $\sigma$, in the medium diversity - high flexibility scenario. The most suitable values are $\omega^{*}=0.7, \alpha^{*}=0.1$ and $\sigma^{*}=0.2$. In contrast with the EV cases, randomization of the non-linear prices brings additional benefits for the reason explained in Section IV.

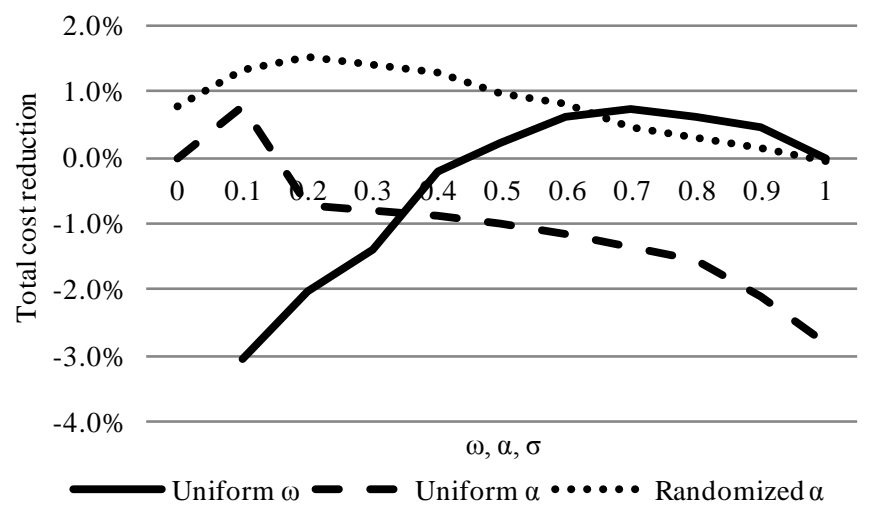

Fig. 5. Benefits of demand response concentration measures for different values of $\omega, \alpha$ and $\sigma$ in medium diversity - high flexibility WA scenario

Fig. 6-7 illustrate the inflexible active demand profile of the microgrid, along with the respective total demand profile for the cases without demand concentration measures, with the uniform flexibility restriction measure, and with the uniform and randomized non-linear pricing measures (applied with the most suitable values of their parameters $\omega^{*}, \alpha^{*}$ and $\sigma^{*}$ ). Fig. 6 refers to the high diversity - high flexibility scenario, while Fig. 7 refers to the low diversity - high flexibility scenario.

In line with the discussion in Section IV, the uniform flexibility restriction and non-linear pricing approaches achieve a relatively effective flattening of the total demand profile in the high diversity scenario, and the additional benefit of rando mization is relatively s mall, yet still visible.

The results are significantly different in the low diversity scenario. Given that all WA are identical, the new peak created when no measures are taken is even larger, and the uniform flexibility restriction approach cannot improve at all the obtained solution ("No measure" and "Uniform $\omega$ " curves coincide). Due to the effect of differentiated price variations at the different nodes analyzed in Section IV-B, uniform nonlinear pricing reduces the size of the new demand peak. Randomized non-linear pricing constitutes the only measure than flattens sufficiently the total demand profile and its benefit is significantly higher than in the low diversity case.

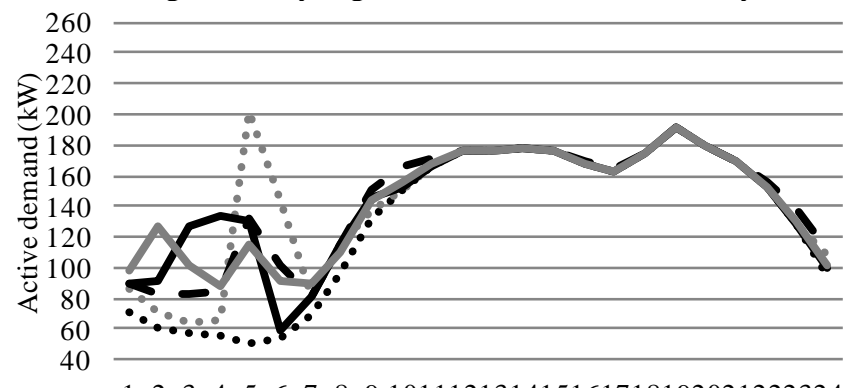

$\begin{array}{llllllllll}12 & 3 & 5 & 6 & 7 & 8 & 9 & 101112131415161718192021222324\end{array}$ Hour

$\ldots .$. Inflexible demand $\cdots .$. No measure $\quad-$ Uniform $\omega$ Uniform $\alpha$ Randomized $\alpha$

Fig. 6. Active demand profile of microgrid in high diversity - high flexibility WA scenario

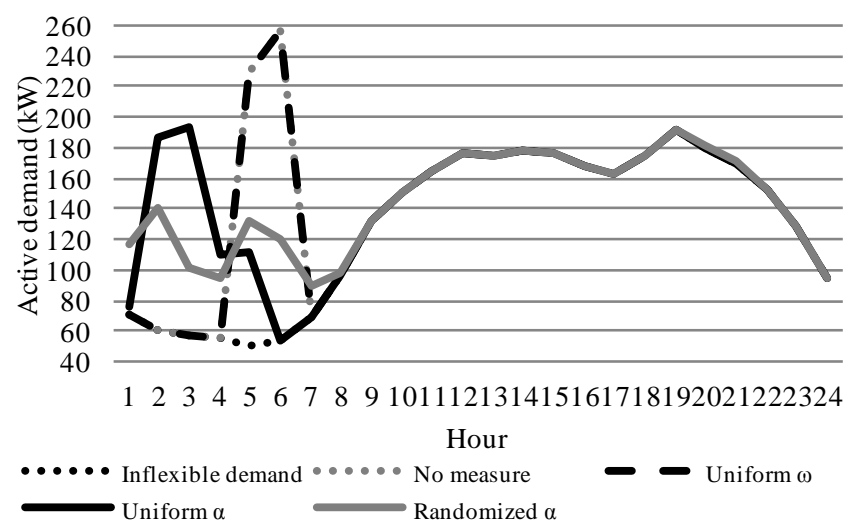

Fig. 7. Active demand profile of microgrid in low diversity - high flexibility WA scenario

For each diversity scenario, as the flexibility of WA is enhanced, the size and the cost implications of the new peaks created in the case without measures are significantly aggravated, as more WA can execute their cycles at the periods with the lowest linear prices; as a result, the benefits of the three measures are increased (Table V). For each fle xibility scenario, in line with the findings from Fig. 6-7, as the diversity of WA is reduced, the benefit of non-linear prices' randomization is enhanced, and the most suitable value of the standard deviation is increased.

TABLE V

OPTIMAL TUNING AND BENEFTS OF DEMAND RESPONSE CONCENTRATION MEASURES IN WA SCENARIOS

\begin{tabular}{|l|c|c|c|c|c|c|}
\hline Scenario & $\begin{array}{c}\text { Uniform flexibility } \\
\text { restriction }\end{array}$ & \multicolumn{2}{|c|}{$\begin{array}{c}\text { Uniform non-linear } \\
\text { pricing }\end{array}$} & $\begin{array}{c}\text { Randomize dnon- } \\
\text { linear pricing }\end{array}$ \\
\hline & $\omega^{*}$ & Benefit & $\alpha^{*}$ & Benefit & $\sigma^{*}$ & Benefit \\
\hline LD-LF & 1 & $0.00 \%$ & 0 & $0.00 \%$ & 0.5 & $1.01 \%$ \\
\hline LD-MF. & 1 & $0.00 \%$ & 0.1 & $2.77 \%$ & 0.3 & $4.35 \%$ \\
\hline LD-HF. & 1 & $0.00 \%$ & 0.1 & $2.77 \%$ & 0.3 & $4.35 \%$ \\
\hline MD-LF & 1 & $0.00 \%$ & 0.1 & $0.08 \%$ & 0 & $0.08 \%$ \\
\hline MD-MF & 1 & $0.00 \%$ & 0 & $0.18 \%$ & 0.2 & $0.31 \%$ \\
\hline MD-HF & 0.7 & $0.71 \%$ & 0.1 & $0.78 \%$ & 0.2 & $1.51 \%$ \\
\hline HD-LF & 1 & $0.00 \%$ & 0.1 & $0.03 \%$ & 0 & $0.03 \%$ \\
\hline HD-MF & 1 & $0.00 \%$ & 0 & $0.04 \%$ & 0.1 & $0.14 \%$ \\
\hline HD-HF & 0.7 & $0.80 \%$ & 0.1 & $0.94 \%$ & 0.1 & $1.17 \%$ \\
\hline
\end{tabular}




\section{D.Analysis of Cases with Flexible EV and Flexible WA}

As discussed in Section IV-D, in cases where the microgrid includes both loads with continuously adjustable power levels and loads with deferrable cycles, either identical or differentiated between the different types, $\omega, \alpha$ and $\sigma$ values can be applied. Tables VI and VII present the most suitable values of the parameters of the three measures, as well as their benefits, for each of these two approaches and for different scenarios with both EV and WA in the microgrid. The second approach expands the space of the heuristic search for high quality solutions and thus leads to higher benefits in every scenario and for each of the three measures. However, these higher benefits come with the higher complexity in tuning the differentiated parameters for the different load types.

TABLE VI

OPTIMAL IDENTICAL TUNING AND BENEFTS OF DEMAND RESPONSE CONCENTRATION MEASURES IN EV-WA SCENARIOS

\begin{tabular}{|l|c|c|c|c|c|c|}
\hline Scenario & \multicolumn{2}{|c|}{$\begin{array}{c}\text { Uniform flexi bility } \\
\text { restriction }\end{array}$} & \multicolumn{2}{|c|}{$\begin{array}{c}\text { Uniform non-linear } \\
\text { pricing }\end{array}$} & \multicolumn{2}{c|}{$\begin{array}{c}\text { Randomizednon- } \\
\text { linear pricing }\end{array}$} \\
\hline & $\omega^{*}$ & Benefit & $\alpha^{*}$ & Benefit & $\sigma^{*}$ & Benefit \\
\hline LD-LP-LF & 1 & $0 \%$ & 0 & $0 \%$ & 0.2 & $1.53 \%$ \\
\hline LD-LP-HF & 0.4 & $10.73 \%$ & 0.2 & $11.82 \%$ & 0.3 & $14.93 \%$ \\
\hline LD-HP-LF & 1 & $0 \%$ & 0.8 & $0.03 \%$ & 0.4 & $6.83 \%$ \\
\hline LD-HP-HF & 0.4 & $32.14 \%$ & 0.3 & $36.27 \%$ & 0.3 & $37.92 \%$ \\
\hline HD-LP-LF & 1 & $0 \%$ & 0.1 & $0.02 \%$ & 0 & $0.02 \%$ \\
\hline HD-LP-HF & 0.5 & $4.46 \%$ & 0.1 & $4.83 \%$ & 0.1 & $5.18 \%$ \\
\hline HD-HP-LF & 1 & $0 \%$ & 0 & $0 \%$ & 0 & $0 \%$ \\
\hline HD-HP-HF & 0.2 & $31.57 \%$ & 0.4 & $31.90 \%$ & 0.3 & $32.20 \%$ \\
\hline
\end{tabular}

TABLE VII

OPTIMAL DIFFERENTIATED TUNING AND BENEFITS OF DEMAND RESPONSE CONCENTRATION MEASURES IN EV-WA SCENARIOS

\begin{tabular}{|l|c|c|c|c|c|c|c|c|c|}
\hline Scenario & \multicolumn{3}{|c|}{$\begin{array}{c}\text { Uniform flexibility } \\
\text { restriction }\end{array}$} & \multicolumn{3}{c|}{$\begin{array}{c}\text { Uniform non-linear } \\
\text { pricing }\end{array}$} & \multicolumn{3}{c|}{$\begin{array}{c}\text { Randomizednon- } \\
\text { linear pricing }\end{array}$} \\
\hline & $\omega_{E V}^{*}$ & $\omega_{W A}^{*}$ & Benefit & $\alpha_{E V}^{*}$ & $\alpha_{W A}^{*}$ & Benefit & $\sigma_{E V}^{*}$ & $\sigma_{W A}^{*}$ & Benefit \\
\hline LD-LP-LF & 0.8 & 1 & $0.12 \%$ & 1.8 & 0 & $0.15 \%$ & 0 & 1.3 & $1.62 \%$ \\
\hline LD-LP-HF & 0.7 & 0.5 & $11.80 \%$ & 0 & 0.2 & $12.10 \%$ & 0.1 & 0.4 & $15.01 \%$ \\
\hline LD-HP-LF & 0.6 & 1 & $5.80 \%$ & 2 & 0 & $5.84 \%$ & 0 & 1.5 & $8.42 \%$ \\
\hline LD-HP-HF & 0.5 & 0.4 & $35.56 \%$ & 0.1 & 0.3 & $36.56 \%$ & 0.1 & 0.5 & $38.16 \%$ \\
\hline HD-LP-LF & 1 & 1 & $0 \%$ & 0.1 & 0.1 & $0.02 \%$ & 0 & 0 & $0.02 \%$ \\
\hline HD-LP-HF & 0.2 & 0.6 & $5.07 \%$ & 1.2 & 0.1 & $5.09 \%$ & 0 & 0.2 & $5.32 \%$ \\
\hline HD-HP-LF & 1 & 1 & $0 \%$ & 0.9 & 0.1 & $0.04 \%$ & 0 & 0 & $0.04 \%$ \\
\hline HD-HP-HF & 0.2 & 0.6 & $32.51 \%$ & 1.9 & 0.1 & $32.58 \%$ & 0 & 0.3 & $32.66 \%$ \\
\hline
\end{tabular}

\section{CONCLUSIONS AND FUTURE WORK}

This paper proposes a novel, fully price-based measure to tackle flexible loads' response concentration in distributed management of DER within microgrids. Apart from the traditional linear prices, a non-linear price signal is transmitted to these loads, penalizing the extent of flexibility utilized. This signal is customized to the operating properties of the different flexible load types, by penalizing the square of the demand and the duration of cycle delay for loads with continuously adjustable power levels and deferrable cycles respectively.

This measure not only addresses the acceptability and feasibility limitations of a previously proposed alternative measure imposing flexibility restrictions on the loads, but is also demonstrated to produce more efficient solutions, for both types of loads. For the latter type, a simple approach randomizing the non-linear prices transmitted to the loads is shown to bring additional benefits, especially in cases where the operating diversity of these loads is low.
Future work aims at developing efficient techniques for the optimal tuning of the non-linear and randomized pricing measures' parameters, without extensive computational requirements. Furthermore, uncertainties associated with renewable micro-generators' outputs and consumers' preferences and requirements affecting the operation of flexible loads, will be incorporated in the microgrid management problem and addressed through a stochastic reformu lation of the proposed distributed mechanism.

\section{REFERENCES}

[1] N. Hatziargyriou, H. Asano, R. Iravani, and C. Marnay, "Microgrids," IEEE Power Energy Mag., vol. 5, no. 4, pp. 78-94, Jul.-Aug. 2007.

[2] A. G. Tsikalakis, and N. D. Hatziargyriou, "Centralized control for optimizing Microgrids operation," IEEE Trans. Energy Conv., vol. 23, no. 1, pp. 241-248, Mar. 2008.

[3] D. Pudjianto, P. Mancarella, C. K. Gan, and G. Strbac, "Closed loop price signal based market operation within Microgrids," Eur. Trans. Elec. Power, vol. 21, no. 2, pp. 1310-1326, Mar. 2011

[4] M. S. Bazaraa, H. D. Sherali, and C. M. Chetty, Nonlinear Programming: Theory and Algorithms, 3rd ed. Hoboken, NJ, USA: Wiley, 2006.

[5] A. L. Motto, F. D. Galiana, A. J. Conejo, and M. Huneault, "On walrasian equilibrium for pool-based electricity markets," IEEE Trans. Power Syst., vol. 17, no. 3, pp. 774-781, Aug. 2002.

[6] J. Warrington, P. Goulart, S. Mariethoz, and M. Morari, "A market mechanism for solving multi-period optimal power flow exactly on AC networks with mixed participants," Proc. Amer. Control Conf., Montreal, Canada, 2012.

[7] D. Zelazo, R. Dai, and M. Mesbahi, “An energy management system for off-grid power systems,” Energy Syst., vol. 3, no. 2, pp. 153-179, Jun. 2012.

[8] N. Gatsis, and G. B. Giannakis, "Residential load control: distributed scheduling and convergence with lost AMI messages," IEEE Trans. Smart Grid, vol. 3, no. 2, pp. 770-786, Jun. 2012.

[9] A. Nedich, and A. Ozdaglar, "Approximate primal solutions and rate analysis for dual subgradient methods," SIAM Jour. Optim., vol. 19, no. 4, pp. 1757-1780, Dec. 2008.

[10] D. Papadaskalopoulos, D. Pudjianto, and G. Strbac, "Decentralized coordination of microgrids with flexible demand and energy storage," IEEE Trans. Sust. Energy, vol. 5, no. 4, pp. 1406-1414, Oct. 2014.

[11] D. Papadaskalopoulos, and G. Strbac, "Decentralized optimization of flexible loads operation in electricity markets," Proc. PowerTech Conf., Grenoble, France, 2013.

[12] A. Borghetti, A. Frangioni, F. Lacalandra and C. A. Nucci, "Lagrangian Heuristics Based on Disaggregated Bundle Methods for Hydrothermal Unit Commitment," IEEE Trans. Power Syst, vol. 18, no. 1, pp. 313323, Feb. 2003

[13] J. A. P. Lopes, F. J. Soares, and P. M. R. Almeida, "Integration of electric vehicles in the electric power system," IEEE Proc., vol. 99, no. 1, pp. 168-183, Jan. 2011.

[14] R. Stamminger, Synergy Potential of Smart Domestic Appliances in Renewable Energy Systems, ed. University of Bohn, Shaker Verlag, Aachen, 2009.

[15] A. H. Mohsenian-Rad, and A. Leon-Garcia, "Optimal residential load control with price prediction in real-time electricity pricing environments," IEEE Trans. Smart Grid, vol. 1, no. 2, pp. 120-133, Sep. 2010.

[16] S. Papathanassiou, Study case LV network, 2003. [Online]. Available: microgrids.power.ece.nt ua.gr/document s/StudyCase\%20LVNet work.pdf

Dimitrios Papadaskalopoulos (M'13) is a Research Associate at Imperial College London, London, U.K., since 2013. His current research interests include demand side and storage participation in power system operation and planning, electricity markets modeling and pricing, and active management of distribution net works.

Goran Strbac (M'95) is a Professor of Electrical Energy Systems at Imperial College London, London, U.K., since 2005. His research interests include electricity system operation, investment and pricing, and integration of renewable generation and distributed energy resources. 